\title{
Design of Experiments Teaching on Inversion Layer Turbulent Movement Simulation in Glass Water Tank
}

\author{
Xiaohang Wen, Lei Zhao, Binyun Wang \\ School of Atmospheric Sciences, Chengdu University of Information Technology, Chengdu, China \\ Email:wxh@cuit.edu.cn
}

How to cite this paper: Wen, X. H., Zhao, L., \& Wang, B. Y. (2020). Design of Experiments Teaching on Inversion Layer Turbulent Movement Simulation in Glass Water Tank. Open Journal of Social Sciences, 8, 250-259.

https://doi.org/10.4236/jss.2020.84018

Received: January 2, 2020

Accepted: April 14, 2020

Published: April 17, 2020

Copyright $\odot 2020$ by author(s) and Scientific Research Publishing Inc. This work is licensed under the Creative Commons Attribution International License (CC BY 4.0).

http://creativecommons.org/licenses/by/4.0/

(c) (i) Open Access

\begin{abstract}
The glass water tank experiment is using water as the medium to simulate the temperature fields' structure characteristics and development process of the convection boundary layer in the laboratory. This experiment uses a fast-responding temperature sensor to measure vertical profile of temperature and temperature differences at different height. In this experiment, parameters such as tropospheric boundary thickness and stability are obtained. The experimental results show that the indoor water tank convection system can simulate the formation, development and extinction of inversion layer in boundary layer. This experiment is suitable as a professional experiment for atmospheric fluid dynamic course. Meanwhile, students' understanding of meteorological concepts and basic principles is more profound and excellent teaching effect is achieved, and thus the designed experiments have certain promotion value.
\end{abstract}

\section{Keywords}

Convection Water Tank, Inversion Layer, Atmospheric Boundary Layer, Experimental Teaching

\section{Introduction}

Glass water tank simulation experiments can simulate physical phenomena in the atmospheric boundary layer, which helps to understand intuitively the inverse temperature and convective rising activities in the real atmospheric boundary layer. In the glass water tank simulation experiment, it is easy to obtain parameters and statistics which are difficult obtained in field observation experiment. It is easy to observe the meteorological variables such as wind direction, 
wind speed, temperature, and humidity profile in boundary layer. In general, obtaining meteorological elements of the atmospheric boundary layer can only be measured by meteorological tower stations or air balloons, which makes observing the actual boundary layer atmosphere very complicated. So the glass water tank simulation experiment can well connect the atmospheric field observation with the theoretical model (Xu \& Wu, 2008; Yan et al., 2010).

The indoor simulated atmospheric turbulence has the characteristics of convenient control, repeatable simulation conditions, and the flexibility to establish certain real atmospheric scenes, which is widely used. Many scholars have designed different types: such as wind tunnels, convective water tanks, towed water tanks, gas turbulence pools, salt water tanks, etc.) devices, used to simulate the temperature in the turbulent atmosphere, the heat transfer process, gas pollution in the convective mixing, diffusion, etc. Regarding the structure and turbulence characteristics of the convective trough to simulate the convective boundary layer (CBL), the American scholars Willis and Deardorff conducted experiments in this area as early as 1974 (Willis \& Deardorff, 1976). Domestic scholars Yuan Renmin and Sun Jianlu conducted research after 2000, and achieved many important results in the temperature field and velocity field structure of the entrainment layer, the characteristics of the turbulence spectrum and the physical mechanism generated (Yan et al., 2010). Atmospheric science is a discipline that studies the laws of atmospheric movement, the causes of weather and climate events, and forecasting and evaluation service technologies. It is characterized by the equal emphasis on theoretical teaching and practical teaching. Traditional atmospheric science experimental teaching is generally completed in a laboratory or at a meteorological observatory, and is mainly based on teacher lectures and demonstrations. Due to space and time constraints, teachers often cannot fully display weather and climatic phenomena for students, resulting in fewer opportunities for students to do it themselves, poorer sense of real situation, and less chance for independent innovation ideas to be verified. Using virtual reality technology for atmospheric science experimental teaching, students can work in real meteorological observation or forecasting environments. They can practice programmatic operation steps according to the teacher's arrangement, and they can also manually verify their experimental hypotheses and repeat them, reduce the cost of experiment consumables or the risk of experiment. According to the characteristics of virtual reality technology, it has good educational value in experimental teaching of atmospheric sciences (Zhang et al., 2017; Wang et al., 2008; Zong \& Ma, 2011; Zhang, et al., 2008).

In this experiment, a glass water tank is used to simulate the development process of the atmospheric boundary layer through water, a fluid medium, and provide a scientific explanation for the physical processes occurring in the atmospheric boundary layer. Compared with traditional atmospheric science field observation experiments, the glass water tank experiment is repeatable and can simulate the inverse temperature process. This experiment belongs to the experimental course of atmospheric fluid mechanics and atmospheric dynamics. The 
purpose is to let students' understanding of the occurrence, development, and extinction of inverse temperature in the atmospheric boundary layer, and to support theoretical teaching (Zong \& Ma, 2011; Zhang et al., 2008; Guan \& Wu, 2016). Therefore, using a glass water tank experiment, the temperature profile of the convective boundary layer and its development process are simulated in the laboratory under the condition of uniform heating of the underlying surface, and the formation, occurrence, development and demise of the inversion layer are reproduced. This experiment is suitable for students majoring in atmospheric sciences and meets the needs of supporting experiments in atmospheric fluid mechanics courses (Zhang et al., 2008; Pettré et al., 2009).

\section{Experiment Introduction}

\subsection{Introduction of Experimental Equipment}

Glass water tanks include automatic temperature-control heating water tanks, intelligent heating water storage tanks, ion-exchange deionized water equipment, diffuse flow control devices, temperature detection systems, motion drive systems, large-caliber laser collimated light generators, CCD cameras, Data acquisition system (including control and management operation software) and other components and modules. During the experiment, hot water and cold water were poured into a tempered glass water tank in advance to make it layered. The oil tank with a built-in heating tube was used to heat the cold water in the water tank and transfer the collected temperature data to a computer to simulate the development of the actual atmospheric boundary layer. The collimated light emitted by the laser generator is used to form a light spot through the glass water tank, and the image is collected by the CCD and transmitted to the computer (Figure 1).

In this experiment, the conditions of dynamic similarity, thermal similarity,

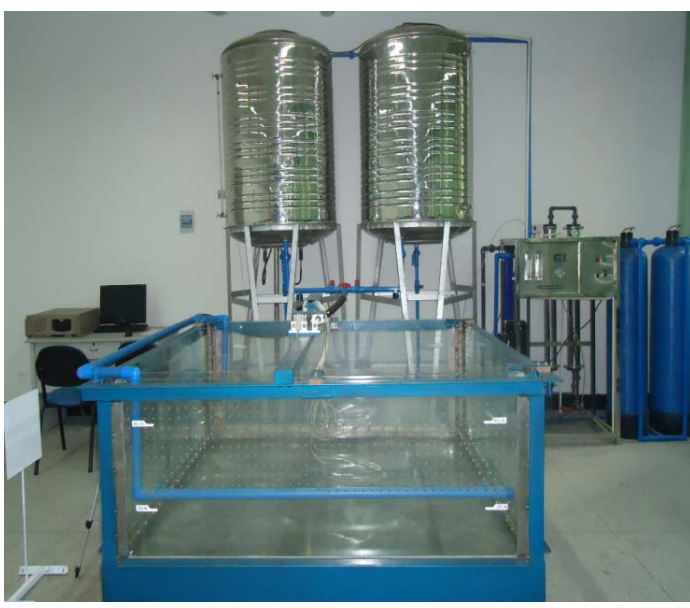

(a)

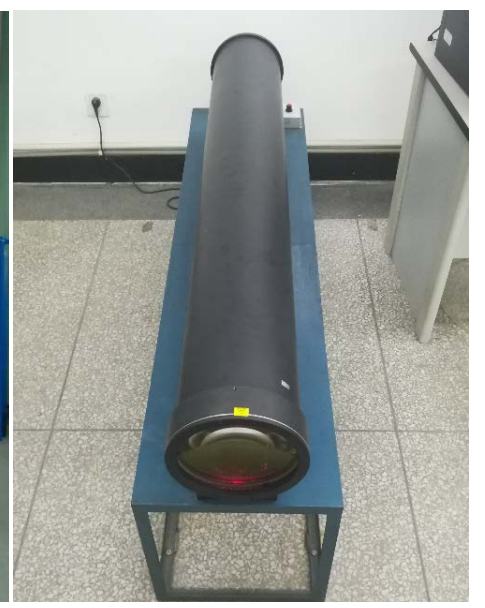

(b)

Figure 1. Glass water tank system of the Atmospheric Simulation Laboratory of Chengdu University of Information Technology. (a) Glass water tank and water purifier systems, (b) Collimated laser scintillator. 
geometrical similarity, and similar motion are fully considered. The bottom of the glass sink is a metal plate, and $10 \mathrm{~mm}$ thick transparent glass forms the edge of the sink. The size of the sink is $150 \mathrm{~cm} \times 150 \mathrm{~cm} \times 60 \mathrm{~cm}$, which meets the requirements of geometric similarity (Yan et al., 2010), and the need for indoor simulation is considered. A metal plate with a size of $145 \mathrm{~cm} \times 145 \mathrm{~cm} \times 6 \mathrm{~cm}$ is placed at the bottom of the sink for heating the fuel tank, (Figure 1). The oil tank is full of transformer oil. The advantages are high margin performance and small thermal expansion coefficient. Electric heating tubes are evenly placed in the transformer oil. Oil is added to the electric heating tubes. After the oil heats up, the water in the water tank is heated to make the bottom of the water tank uniform. heating. The heating power of the fuel tank is adjustable and the maximum heating power is $35 \mathrm{~kW}$.

\subsection{Principle of Vertical Temperature Profile Measurement}

The temperature vertical profile is measured by installing temperature sensors of different layers in the water tank, and the temperature sensor is a customized platinum resistance thermometer. Because there is a very clear correspondence between platinum resistance and temperature (significant linear relationship) and its chemical properties are stable, platinum resistance is very suitable for measuring temperature.

\subsection{Heat Flux Profile Measurement}

Multiple temperature probes ( 8 in the horizontal direction and 7 in the vertical direction) are used for measurement, and the distance between each probe is about $5 \mathrm{~cm}$. The collected temperature output signal is $25 \mathrm{~Hz}$. After being converted by the $\mathrm{ADC}$ module, it is stored in the computer for processing. The temperature sensor can be driven by a stepper motor and move horizontally. From the measurement results of the temperature profile, meteorological features can be analyzed, such as the convective velocity, heat flux profile, convective boundary layer development, and extinction characteristics. Figure 2 is a schematic diagram of a temperature detector.

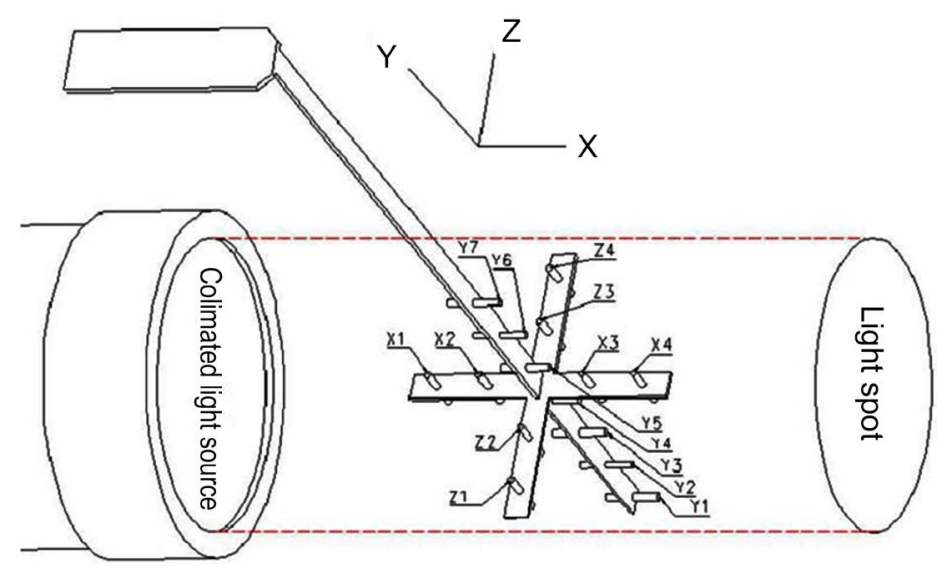

Figure 2. Schematic diagram of temperature detector, $\mathrm{X}$ and $\mathrm{Z}$ are horizontal, $\mathrm{Y}$ is vertical. 


\subsection{Measurement of Turbulent Motion}

The measurement of turbulent motion is based on a large-caliber collimated laser scintillator (Han et al., 2006; Pingyang et al., 2003). After the laser beam is expanded twice, it becomes a collimated light with a diameter of $20 \mathrm{~cm}$. When the collimated light is emitted from the side of the water tank, after passing through the turbulent field in the water tank, a light spot is formed on the other side. We placed a white receiving screen on the other side of the water tank, and used a CCD camera to continuously capture light spot images on the receiving screen, and the images were collected by a computer.

\section{Experimental Design and Experimental Steps}

The experimental design follows the CDIO engineering education model. It focuses on the students and intuitively displays the complex actual atmospheric boundary layer temperature inversion phenomenon in the laboratory sink experiment to cultivate students' understanding and understanding of atmospheric phenomena in atmospheric science (Han et al., 2006; Berggren et al., 2003; Kang et al., 2008; Fan et al., 2011).

The purpose of experimental teaching is to cultivate students' practical skills. The mastery of practical skills can be divided into four stages: operation orientation, imitation, integration and proficiency. It requires students to explore and gradually clarify the experimental content and experimental steps in the actual operation process. In traditional teaching, students will feel that the theoretical and formula parts of the textbook are very boring, and some scenes are even more difficult to describe in language. The actual experimental hands-on operation can transform theoretical teaching into rich practical teaching and increase students' interest in learning. In this experiment, we can follow next step:

Step 1: we let students debug the laser by themselves, turn on the water tank switch and put in cold water heating and other steps to make the students familiar with the water inlet and heating process of the water tank.

Step 2: After the water in the water storage tank is heated, the students themselves put cold water and hot water in the sink separately. The teacher turns on the computer, controls the temperature detector to move in the water tank, and records the data.

Step 3: use the camera to record the image of the light spot formed by the laser through the interface of the cold water and the hot water layer, and explain to the students the principle of light spot formation.

Step 4: Use the recorded temperature data to draw the profile of the boundary layer of the sink, and explain to the students the differences in temperature profiles of different layers.

\subsection{Preview before Class}

At this stage, students are trained to learn and master the process of the formation of the "atmospheric inversion layer". The specific tasks are: 
1) Learn relevant knowledge of the atmospheric boundary layer, understand the height of the atmospheric boundary layer, turbulent structure, temperature layer, and discuss the characteristics that affect the air movement of the boundary layer.

2) Learn the forces on the air movement of the atmospheric boundary layer, and discuss the effects of different underlying conditions (such as different terrain, landforms, buildings and vegetation) on the atmospheric boundary layer.

3) Understand the characteristics of the Ekman layer in the atmospheric boundary layer, and discuss the effects of viscous force, pressure gradient force, and Coriolis force.

4) The types of inversion layer include radiation inversion, sinking inversion, turbulent inversion and advection inversion. Discuss the differences between different types of inversion.

\subsection{Experimental Steps}

In the experiment, the water in the water tank is deionized and purified water. First turn on the reverse osmosis pure water system and fill the cold and hot water storage tanks with water. Pure water is produced by reverse osmosis water purification system, quartz sand filtration system, activated carbon filtration and adsorption technology. Water production is 0.5 tons/hour. After opening the water inlet valve, when the water pressure gauge is greater than $0.1 \mathrm{MPa}$, the production of pure water begins, and the hot and cold water storage tanks are connected by the water guide pipe. Observe the water storage tank level gauge. When the liquid level reaches half the volume of the water storage tank, close the water production system, and the water storage is completed. During operation, a plastic plate with a thickness of about $0.5 \mathrm{~cm}$ was placed around the inside of the glass of the sink, and hundreds of small holes were evenly distributed on the plastic plate. A cold/hot water pipe is placed along the inside of the glass of the sink, and there are openings every $5 \mathrm{~cm}$. The openings face the glass. When the water comes out of the small hole of the cold/hot water pipe, it reaches the open plastic plate, and slowly flows into the glass sink through the small hole of the plastic plate.

Then turn on the computer simulation system, set the heating temperature of the hot water storage tank, and heat the hot water for the test. (Ensure that the storage tank has been filled with a sufficient amount of water when heating, and it is strictly prohibited to heat it without water). When the temperature of the hot water storage tank reaches 40 degrees, stop heating. Before opening the water outlet valve, first open the cold water valve, first add cold water with a depth of about $20 \mathrm{~cm}$ (to the lower edge of the diffuser pipe), close the cold water valve, then open the hot water valve, and add about $25 \mathrm{~cm}$ of hot water. A stable and uniform inversion layer is formed. Finally, the large-caliber laser collimation light generator was finally opened, and the fuel tank was started to be heated. By the heating effect of the fuel tank, the cold water layer closest to the bottom of the water tank began to be heated, simulating the actual atmospheric boundary 
layer change. Click the computer auto-run button, the system will start the simulation experiment and automatically record the data.

\section{Experimental Results}

\subsection{Experimental Process}

After the experiment started, the cold water layer in the water tank was at the bottom and the hot water layer was at the top. At this time, a stable inversion layer was formed. It can be seen from Figure 3(a) that the light spot has obvious layering, the upper layer is an image of turbulent activity in the hot water layer, and the lower part is a static image when the turbulence in the cold water layer is not active. There is a clear dividing line between the two layers. When the cold water at the bottom of the water tank is heated for a period of time, the bottom of the light spot projected on the receiving screen appears ripples (Figure 3(b)). When the fuel tank is heated for 20 minutes, the hot water convection activity at the bottom intensifies, and gradually moves upward to reach the inversion layer interface, and gradually extends from the convection activity to the upper layer (Figure 3(c)).

The temperature probe measured the vertical temperature profile and calculated the heat flux. With the increase of the water storage height in the water tank, the heat flux decreases linearly. After reaching a certain height, the heat flux becomes 0 , continues to become negative, and finally becomes 0 . The structure of the boundary layer is determined by changes in the heat flux profile. The mixed layer is defined as the part where the ground heat flux is positive, and the entrainment layer is the area where the heat flux is negative in the actual atmosphere. The thickness of the atmospheric boundary layer is determined by the height at the maximum negative value of the heat flux profile. The boundary layer thickness $(\mathrm{Z})$, convection velocity $(\mathrm{w})$, temperature $(\mathrm{T})$, and atmospheric stability $(\mathrm{R})$ are finally calculated from the heat flux profile.

After the formation of a stable inversion layer, the oil tank was heated, and the

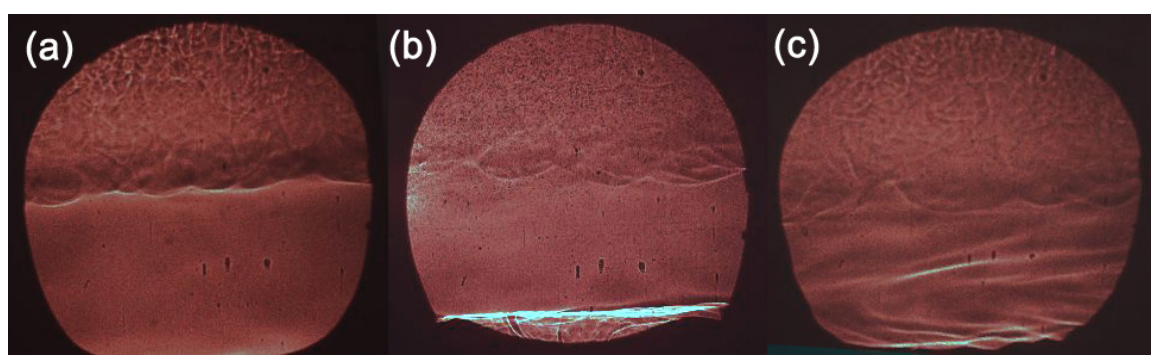

Figure 3. The spot image of the collimated laser transmitter on the receiving screen. (a) Initial stage: the upper part is the hot water layer, and the lower part is the cold water layer, which indicates a stable temperature inversion layer; (b) Heating stage: After the oil tank at the bottom of the water tank is heated for 10 minutes, there are obvious ripples at the bottom, indicating the bottom convection movement Occurs; (c) mixing stage: after the fuel tank is heated for 20 minutes, the convective motion at the bottom rises to the inversion layer, the mixing layer is formed, and the inversion phenomenon is about to end. 
temperature change curve measured by the temperature probe was measured over time. As the bottom of the glass water tank heats up, thermal convection begins to develop, and the temperature in the lower part gradually rises, corresponding to the inverse temperature phenomenon of the actual atmospheric boundary layer. The temperature measured by the upper temperature probe gradually decreased. After the fuel tank was heated for 20 minutes, the inversion layer disappeared, and the overall water temperature in the water tank was about $34^{\circ} \mathrm{C}$. The heat flux profile can be calculated from the temperature profile, and the heat flux also decreases linearly with increasing height until it becomes zero at a certain height. The purpose of this experiment is to simulate the thickness of the boundary layer in the laboratory and calculate some characteristic parameters of the boundary layer, which can be verified in real atmospheric sounding experiments.

\subsection{After Class Discussion}

The theoretical knowledge of the atmospheric boundary layer before the experiment only explains the principle of inverse temperature formation, and it cannot fully clarify the experimental phenomena and the laws of inverse temperature development at each stage. After this experiment, students should analyze and discuss the experimental phenomena after class. Contains the following:

1) Analyze the role of the temperature decrease rate $\gamma$ in the formation of the inversion layer.

2) After the formation of the inversion layer, the relationship between the temperature layer inside the boundary layer and the stability of the atmosphere is analyzed.

2) Discuss the harm caused by the formation of the inversion layer, and give examples of major inversion events in countries around the world.

3) Record experimental phenomena and write experimental reports.

\section{Summary and Discussion}

The emergence of the inversion layer in the atmospheric boundary layer can hinder air convection and exacerbate air pollution near the ground. In this experiment, the inversion process in the atmospheric boundary layer is simulated by a water tank, and the structure of the temperature field in the inversion layer is analyzed by temperature probes and optical measurements. The law of transfer of air in the boundary layer is an experimental course in the theoretical courses of "Atmospheric Boundary Layer" and "Atmospheric Dynamics" to cultivate students to grasp the laws and phenomena of atmospheric movements that commonly exist in the atmospheric boundary layer, which not only enhances the depth and breadth of the experiment, reproduces the complex inverse temperature phenomena in the real atmosphere in the laboratory, but also strengthens students' understanding of the atmosphere. The understanding of complex formulas of fluids and dynamics has certain reference significance for other con- 
firmatory experimental teaching reforms.

From the results of the experiment, it can be found that after the cold water and the hot water are mixed, the hot water layer is stably maintained on the cold water layer for a period of time, and an obvious layer is formed. This shows that there is also a phenomenon in the actual atmosphere: stable cold air is maintained under the hot air and continues for a long time without rapid diffusion. It can be seen from the temperature profile that the temperature increases with height. This will help to form a deep temperature inversion layer and form air pollution. The experimental results have good teaching and practical significance for students to understand the dynamic and thermal mechanisms of the atmospheric boundary layer and the appearance, formation and disappearance of the inversion layer.

With the further development of atmospheric sounding experimental technology, the content, methods and modes of practical training will undergo profound changes. The traditional on- and off-campus experimental models will develop towards a new type of laboratory-practice education mode, which will definitely be applied. The new pattern of training creative and scientific and technological talents will create a new future for the development of modern education.

\section{Acknowledgements}

This work was funded by "Collaboration between industry and school Project of Ministry of Education (201901275001)”.

\section{Conflicts of Interest}

The authors declare no conflicts of interest regarding the publication of this paper.

\section{References}

Berggren, K. F., Brodeur, D., Crawley, E. F., et al. (2003). CDIO: An International Initiative for Reforming Engineering Education. World Transactions on Engineering and Technology Education, 2, 49-52.

Fan, B., Wu, L., Lin, H., et al. (2011). Study of Innovative Experimental Platform Based on CDIO Model. Research and Exploration in Laboratory, 6.

Guan, Z. Y., \& Wu, L. B. (2016). Construction and Reform of Curriculum System for Atmospheric Science Majors. China University Teaching, No. 11, 47-52.

Han, Y., Fan, W., Rao, R., et al. (2006). Experimental Measurement in Atmospheric Aerosol Optical Depth Continuous Spectrum. Chinese Journal of Process Engineering, 6, 32 .

Kang, Q., Lu, X., \& Xiong, G. (2008). On the CDIO Syllabus and the Cultivation of Innovative Talents. Journal of Higher Education Research, 4.

Pettré, J., Ondřej, J., Olivier, A. H., et al. (2009). Experiment-Based Modeling, Simulation and Validation of Interactions between Virtual Walkers. Proceedings of the 2009 ACM SIGGRAPH/Eurographics Symposium on Computer Animation, 189-198.

https://doi.org/10.1145/1599470.1599495 
Pingyang, L., Weimei, J., Jianning, S., et al. (2003). A Laboratory Modeling of the Velocity Field in the Convective Boundary Layer with the Particle Image Velocimetry Technique. Advances in Atmospheric Sciences, 20, 631. https://doi.org/10.1007/BF02915506

Wang, D. H., Liu, C. J., \& Liu, Y. (2008). A Preliminary Analysis of Features and Causes of the Snow Storm Event over the Southern China in January 2008. Acta Meteorologica Sinica, No. 3, 405-422.

Willis, G. E., \& Deardorff, J. W. (1976). A Laboratory Model of Diffusion into the Convective Planetary Boundary Layer. Quarterly Journal of the Royal Meteorological Society, 102, 427-445. https://doi.org/10.1002/qj.49710243212

Xu, A. L., \& Wu, X. Q. (2008). Laboratory Tank Simulation on Stable Boundary Layer and Convective Boundery layer. Journal of Atmospheric and Environmental Optics, 3, 105-110.

Yan, R. M., Wu, X. P., \& Luo, T. (2010). A Water Tank Study of Horizontal Temperature Characteristics of the Convective Boundary Layer. Journal of University of Science and Technology of China, 40, 9-14.

Zhang, D. G., Wang, H., \& Cui, Y. Q. (2017). Analysis of Atmospheric Boundary Layer Inversion Characteristics Based on Microwave Radiometer Observation in Ji'nan in 2015. Journal of Arid Meteorology, 35, 43-50.

Zhang, W. Y., Tong, J. L., \& Song, J. Y. (2008). Discussion on Teaching Contents and Methods of Atmospheric Exploration Experiment Practice. Higher Education of Sciences, 1, 58-60.

Zong, Z. P., \& Ma, J. (2011). The Relationship between the Strength Variablity of Freezing Rain and the Character of Inversion in the Beginning of 2008. Meteorological Monthly, 37, 156-160. 